\title{
DAPT Score to Stratify Ischemic and Bleeding Risk after Percutaneous Coronary Intervention: An Updated Systematic Review, Meta-Analysis, and Meta-Regression of 100,211 Patients
}

\author{
Claudio Montalto ${ }^{1,2}$ Marco Ferlini ${ }^{1}$ Matteo Casula ${ }^{1}$ Alessandro Mandurino-Mirizzi ${ }^{1}$ \\ Francesco Costa ${ }^{2}$ Sergio Leonardi ${ }^{3}$ [i] Luigi Oltrona Visconti ${ }^{1}$
}

1 Division of Cardiology, Fondazione IRCCS Policlinico San Matteo,
Pavia, Italy
2Department of Clinical and Experimental Medicine, Policlinic “G.
Martino," University of Messina, Messina, Italy
${ }^{3}$ Department of Molecular Medicine, University of Pavia, Pavia, Italy

Thromb Haemost 2021;121:687-689.
Address for correspondence Marco Ferlini, MD, Division of Cardiology, Fondazione IRCCS Policlinico San Matteo, Viale Camillo Golgi 19, 27100 Pavia, Italy (e-mail: marco.ferlini@gmail.com).

sion we assessed the effect of a longer uneventful run-in DAPT period before DS calculation on the score's ability to predict ischemic and bleeding events. Treatment effect is reported as relative risk (RR) and 95\% confidence interval (CI). Statistical analysis was performed using R environment.

Overall, nine studies were included in our random-effect meta-analysis. ${ }^{2,6-13}$ Their characteristics are summarized in - Supplementary Table $\mathbf{S 1}$ (available in the online version). Of the 100,211 patients included (-Supplementary Table S2, available in the online version), $42.5 \%$ had a DS $\geq 2$ and when compared with those with lower DS, they experienced a significantly higher hazard of MI and ST combined (RR: 1.72; 95\% CI: $1.50-1.97 ; p<0.0001)$ and a significantly lower hazard of bleeding (RR: 0.79; 95\% CI: 0.70-0.89; $p=0.0001$; - Fig. 2). These findings were confirmed by our secondary analysis including only RCTs (RR: 1.86; 95\% CI: 1.45-2.39; $p<0.0001$ for ischemic events; RR: $0.68 ; 95 \% \mathrm{CI}: 0.55-0.84 ; p=0.0004$ for bleedings; - Supplementary Fig. S2A [available in the online version]) or only registries (RR: 1.57 ; $95 \% \mathrm{CI}$ : $1.43-2.43$; $p<0.0001$ for ischemic events; RR: 0.85 ; $95 \% \mathrm{CI}$ : $0.74-0.98$; $p=0.03$ for bleedings; - Supplementary Fig. S2B [available in the online version]). These results were confirmed also by excluding the derivation cohort from the analysis (-Supplementary Fig. S2C [available in the online version]).

Only four studies separately reported outcomes of subjects according to DAPT duration and DS strata: in this secondary analysis of 27,462 patients, in the DS $\geq 2$ stratum prolonged DAPT was associated with a significantly lower occurrence of MI and ST combined (RR: 0.54; 95\% CI: 0.43-0.67; $p<0.0001$ ) without a significant increase in bleeding (RR: 1.26 ; $95 \% \mathrm{CI}$ : received

August 20, 2020

accepted after revision

October 15, 2020

published online

November 19, 2020 (c) 2020. Thieme. All rights reserved. Georg Thieme Verlag KG,

Rüdigerstraße 14,

70469 Stuttgart, Germany
DOI https://doi.org/ 10.1055/s-0040-1721145. ISSN 0340-6245. 


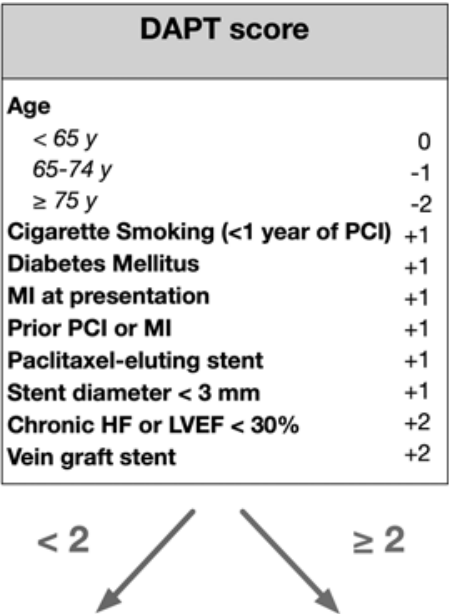

\section{Low DAPT score}

Prolonged DAPT not recommended

\section{High DAPT score}

Prolonged DAPT recommended

Fig. 1 Variables included in the DAPT score. ${ }^{2}$ DAPT, dual antiplatelet therapy.

A

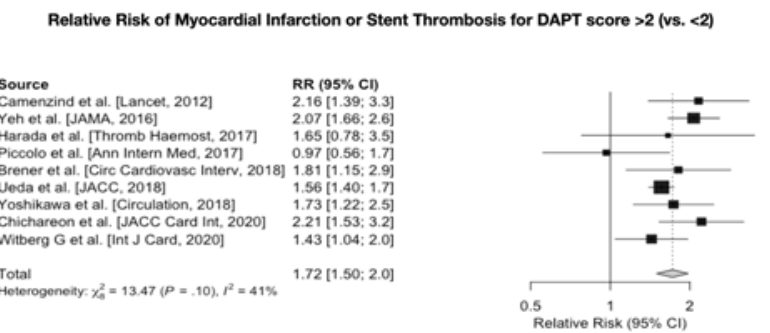

B

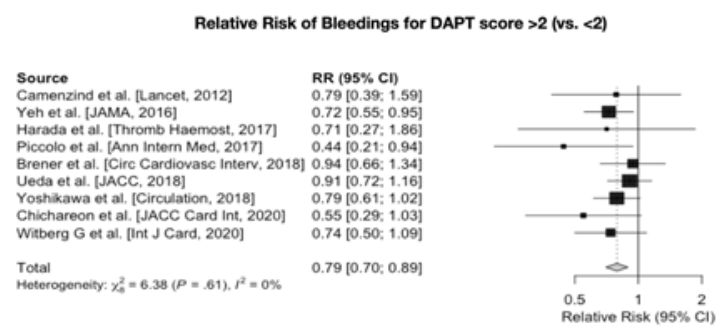

Fig. $2(A, B)$ Forest plot of the main analysis. Random-effect relative risks (RRs) are shown.

0.89-1.76; $p=0.19)$ when compared with standard DAPT duration. (-Supplementary Fig. $\mathbf{5 3}$ [available in the online version ]) On the contrary, in the low DS stratum prolonged DAPT was associated with an increased occurrence of bleedings (RR: 2.00; 95\% CI: 1.49-2.70; $p<0.001$ ) and a neutral effect on ischemic events (RR: $1.03 ; 95 \% \mathrm{Cl}$ : 0.65-1.61; $p=0.9$ ).

Our analysis revealed that the DS had only a modest discriminative power in predicting ischemic (pooled $c$-stat: $0.65 ; 95 \% \mathrm{CI}$ : $0.62-0.69$ ) and bleeding events (pooled $c$-stat: 0.66; 95\% CI: 0.63-0.69; - Supplementary Fig. S4A and - Supplementary Table S3 [available in the online version]). Calibration was suboptimal with a tendency toward underpredicting both ischemic (pooled O:E ratio: 0.48; 95\% CI: 0.31-0.76) and bleeding events (pooled O:E ratio: 0.65, 95\% CI: 0.36-1.14; -Supplementary Fig. S4B and -Supplementary Table S3 [available in the online version]). Finally, in the framework of a meta-regression analysis, we observed that a longer run-in period of DAPT before DS calculation significantly increased the RR of ischemic events $(p<0.0001)$ and significantly decreased the RR of bleedings $(p=0.0003)$ in the DS $\geq 2$ stratum (-Supplementary Fig. S5 [available in the online version]) and was associated with a significant increase in the $c$-stat for both ischemic and bleeding events (both $p<0.0001$; -Supplementary Fig. S6 [available in the online version]).

The DS was validated in a $\mathrm{RCT}^{6}$ with disappointing results, while a recent meta-analysis showed modest clinical utility for bleeding and ischemic risk stratification. ${ }^{3}$ In the current analysis with a larger population of 100,211 subjects after $\mathrm{PCI}$, a DS $\geq 2$ was confirmed to stratify well patients who are at increased risk of coronary ischemic events and relatively low risk of bleeding, despite only modest discriminative power and suboptimal calibration were observed. These results were confirmed in several sensitivity analyses.

Moreover, prolonged DAPT significantly reduced ischemic events without a payoff in terms of more bleedings in the DS $\geq 2$ strata, while only a detrimental effect of increased bleedings was observed with prolonged DAPT in DS $<2$. These findings corroborate the rationale for prolonged DAPT beyond 1 year in such scenario.

Finally, our meta-regression was the first to analyze the effect of DS calculation at different event-free timeframes from the canonical 12 months and supports the use of the original cut-off at 12 months. In fact, a higher predictive ability both for ischemic and bleeding events was observed and this is probably explained by a longer event-free period after PCI which identifies a relatively low-risk population. Considering that the $c$-stat values for both ischemic and bleeding events showed a significant linear increase with a longer run-in period, 12 months might be considered an adequate time point to calculate the DS.

\section{Limitations}

First, we included data of the original derivation cohort, ${ }^{6}$ but we performed a sensitivity analysis that excluded this population and confirmed our results. Second, validation cohorts included different DAPT types and durations. Third, in the study by Chichareon et al, aspirin was compared with ticagrelor as single antiplatelet therapy. Finally, included studies had different follow-up periods.

In conclusion, the DS is a useful clinical tool able to stratify patients at high residual ischemic risk, relatively low bleeding risk, and who benefit from prolonged DAPT. The DS should be calculated at 12 months after PCI to maximize its discriminative power.

Conflict of Interest

None declared.

\section{References}

1 Valgimigli M, Bueno H, Byrne RA, et al; ESC Scientific Document Group ESC Committee for Practice Guidelines (CPG) ESC National Cardiac Societies. 2017 ESC focused update on dual antiplatelet therapy in coronary artery disease developed in collaboration with EACTS: the task force for dual antiplatelet therapy in 
coronary artery disease of the European Society of Cardiology (ESC) and of the European Association for Cardio-Thoracic Surgery (EACTS). Eur Heart J 2018;39(03):213-260

2 Yeh RW, Secemsky EA, Kereiakes DJ, et al; DAPT Study Investigators. Development and validation of a prediction rule for benefit and harm of dual antiplatelet therapy beyond 1 year after percutaneous coronary intervention. JAMA 2016;315(16):1735-1749

3 Witberg G, Zusman O, Yahav D, Perl L, Vaknin-Assa H, Kornowski R. Meta-analysis of studies examining the external validity of the dual antiplatelet therapy score. Eur Heart J Cardiovasc Pharmacother 2020;6(05):285-291

4 Liberati A, Altman DG, Tetzlaff J, et al. The PRISMA statement for reporting systematic reviews and meta-analyses of studies that evaluate healthcare interventions: explanation and elaboration. BMJ 2009;339:b2700

5 Debray TPA, Damen JAAG, Snell KIE, et al. A guide to systematic review and meta-analysis of prediction model performance. BMJ 2017;356:i6460

6 Camenzind E, Wijns W, Mauri L, et al; PROTECT Steering Committee and Investigators. Stent thrombosis and major clinical events at 3 years after zotarolimus-eluting or sirolimus-eluting coronary stent implantation: a randomised, multicentre, open-label, controlled trial. Lancet 2012;380(9851):1396-1405
7 Piccolo R, Gargiulo G, Franzone A, et al. Use of the dual-antiplatelet therapy score to guide treatment duration after percutaneous coronary intervention. Ann Intern Med 2017;167(01):17-25

8 Harada Y, Michel J, Lohaus R, et al. Validation of the DAPT score in patients randomized to 6 or 12 months clopidogrel after predominantly second-generation drug-eluting stents. Thromb Haemost 2017;117(10):1989-1999

9 Brener SJ, Kirtane AJ, Rinaldi MJ, et al. Prediction of ischemic and bleeding events using the dual antiplatelet therapy score in an unrestricted percutaneous coronary intervention population. Circ Cardiovasc Interv 2018;11(10):e006853

10 Yoshikawa Y, Shiomi H, Watanabe H, et al. Validating utility of dual antiplatelet therapy score in a large pooled cohort from 3 japanese percutaneous coronary intervention studies. Circulation 2018;137(06):551-562

11 Ueda P, Jernberg T, James S, et al. External validation of the DAPT score in a nationwide population. J Am Coll Cardiol 2018;72(10):1069-1078

12 Chichareon P, Modolo R, Kawashima H, et al. DAPT score and the impact of ticagrelor monotherapy during the second year after PCI. JACC Cardiovasc Interv 2020;13(05):634-646

13 Witberg G, Zusman O, Bental T, et al. Validation of the DAPT score in real-world patients undergoing coronary stent implantation. Int J Cardiol 2020;300:99-105 